\title{
Fundamental and effective Yang-Mills vertices
}

\author{
Ralf Hofmann \\ ITP, Universität Heidelberg, Philosophenweg 16, 69120 Heidelberg, Germany
}

\begin{abstract}
.
Calorons and plane waves within and in between them collectively give rise to a thermal ground state. The latter provides a homgeneous energy density and a negative pressure, and it induces quasiparticle masses to part of the propagating spectrum of deconfining SU(2) Yang-Mills thermodynamics (dynamical gauge-symmetry breaking). In the present talk we discuss the role of a single caloron in inducing effective local vertices, characterized by powers of $\hbar$, mediating the interaction of plane waves which propagate over large distances. The constraints on momentum transfers through effective 4-vertices are revisited.
\end{abstract}

Keywords: Calorons, effective gauge coupling, Planck's quantum of action

PACS: $11.10 . \mathrm{Wx}, 11.15 . \mathrm{Tk}, 11.55 . \mathrm{Fv}, 02.60 . \mathrm{Cb}$

\section{INTRODUCTION}

Quantum Yang-Mills theory is a beautiful, successful, and useful concept which, in a largely perturbative interpretation, underlies the present Standard Model of particle physics based on the gauge group $\mathrm{U}(1) \times \mathrm{SU}(2) \times \mathrm{SU}(3)$. Its so-far analysed mathematical implications are impressive and include the construction of all (nonpropagating) selfdual solutions to the 4D Euclidean classical Yang-Mills equations of motion [1, 2, 3, 4], the topological characterization of knots [5] in terms of Wilson-loop expectations in 3D Chern-Simons theory [6] and of differentiable 4D manifolds [7], and investigations on the geometry and dynamics of minimal Yang-Mills solitons [8, 9, 10, 11, 12, 13]. A description of nature in terms of gauge theory, which quantitatively accounts for the ground-state physics, dynamical gauge-symmetry breaking, the (residual) interaction of associated quasiparticles, and the transition to phases with effectively reduced gauge symmetries, requires the combined consideration of plane-wave fluctuations and nonpropagating, selfdual configurations. In a thermodynamic setting, we have generated results in this context [14, 15, 16, 17, 18, 19, 20, 21, 22], for a summary see [23]. A thermodynamical framework is natural since we observe an abundance and overlap of (unresolved) topological solitons of SU(2) Yang-Mills theory (magnetic monopoles) in the thermal ground-state [21] which, in an out-of-thermal-equilibrium situation, leaves little hope for a deterministic description.

In the present talk we elaborate on the correspondence between effective and fundamental field configurations in the deconfining phase of SU(2) Yang-Mills theory, for a discussion of the implications towards low-temperature photon physics see Markus Schwarz' talk [24]. In particular, we will justify the following picture of how the effective theory [14, 15] describes the presence and interplay of particular selfdual field configurations, calorons of topological charge unity, and plane-wave fields: On one hand, those calorons, which in isolation possess vanishing energy-momentum, collectively form an inert and adjoint scalar field $\phi$. This is understood by computing a flat spatial average and a flat average over the caloron scale parameter $\rho$ of an adjointly transforming two-point correlator of the field strength to define the kernel $\mathscr{D}$ of a second-order differential operator which determines $\phi$ 's equation of motion. The observation here is that $\mathscr{D}$ is dominated by calorons with $\rho \sim|\phi|$. Trivial-holonomy calorons [25] exhibit the potential to source and absorb collective plane-wave fluctuations with off-shellness greater than $|\phi|^{2}$ which, in turn, deform these calorons (trivial holonomy $\rightarrow$ small holonomy: short-lived magnetic dipoles [4, 26, 27, 28]). Taking a coarse-grained version of this effect into account, an accurate estimate of the thermal ground-state emerges: Fundamental, collective plane-wave fluctuations contribute in terms of an effective pure-gauge configuration which induces pressure $P_{\mathrm{gs}}$ and energy density $\rho_{\mathrm{gs}}=-P_{\mathrm{gs}}$ linear in temperature $T$. On the other hand, computing the gauge coupling $e$ in the effective theory and, by virtue of $\rho \sim|\phi|^{-1}$ for contributing calorons, using $e$ to determine its action $S_{\rho \sim|\phi|^{-1}}$, the results is $S_{\rho \sim|\phi|^{-1}}=\frac{8 \pi^{2}}{e^{2}}=\hbar$ almost everywhere in the deconfining phase. This suggests that a local Yang-Mills vertex, which, after an appropriate rescaling of the propagating, effective gauge field, carries powers of $\hbar^{1 / 2}$ (3-vertex) or $\hbar$ (4-vertex), is induced by the distortion of isolated, fundamental plane-wave propagations through a single caloron. Thus the calorons within the thermal ground-state lend themselves to induce vertices to the sector of propagating, isolated plane waves. Compared to the contribution of freely propagating, effective plane waves this effect amounts to small radiative corrections 
(collective effect of screened magnetic monopoles generated by dissociation of large-holonomy calorons) [18, 19] in thermodynamical quantities. Since only calorons with $\rho \sim|\phi|^{-1}$ are contributing to the thermal ground state momentum transfers through a vertex harder than $|\phi|^{2}$ are essentially suppressed. As a consequence, no ultraviolet divergences arise in integrating out isolated, fundamental plane-wave fluctuations harder than $|\phi|^{2}$ at resolution $|\phi|$. We stress that the perturbative renormalization programme is in agreement with this observation and that perturbative renormalizablity [29, 30], which states the form invariance of the Yang-Mills action under the process of perturbatively integrating out plane-wave fluctuations that are ultraviolet relative to a given renormalization point $\mu$, is an important guide in the construction of the effective action in the deconfining phase.

\section{EFFECTIVE YANG-MILLS ACTION AND ACTION OF A CONTRIBUTING CALORON}

The spatial coarse-graining over noninteracting trivial-holonomy calorons [25] is performed by determining, in the singular gauge used to construct this caloron in terms of the prepotential of a charge-one instanton [31, 32], the second-order linear operator $D \equiv \partial_{\tau}^{2}+\left(\frac{2 \pi}{\beta}\right)^{2}$ which annihilates the field $\phi[14,15$, 17]: $D \phi=0$. To learn about $D$ in terms of its kernel $\mathscr{K}$, a flat spatial and flat $\rho$ average over a unique, adjointly transforming, scalar two-point correlator of the Yang-Mills field strength needs to be performed [14]. In this process we observe that (i) only magneticmagnetic correlations contribute and that (ii) the $\rho$ integration and thus $\mathscr{K}$ is saturated essentially by contributions around the upper limit $\rho_{c}=|\phi|^{-1}$ since the integral scales as $\rho_{c}^{3}$ [14, 33]. Demanding consistency of $D \phi=0$ with $\phi$ 's first-order Bogmoln'yi equation determines the potential $V(\phi)$ in the effective action. $V(\phi)$ is unique modulo a multiplicative constant: $V(\phi)=\frac{\Lambda^{6}}{\phi^{2}}$. In contrast to perturbation theory, the Yang-Mills scale $\Lambda$ enters here on the level of nonpropagating field configurations as a constant of integration for the following first-order consistency equation [17]

$$
\frac{\partial V\left(|\phi|^{2}\right)}{\partial|\phi|^{2}}=-\frac{V\left(|\phi|^{2}\right)}{|\phi|^{2}}
$$

where $|\phi|$ denotes the space-time independent and gauge invariant modulus of $\phi$. Invoking perturbative renormalizability [29, 30] and the fact that the (nonpropagating) field $\phi$ is inert, one arrives at the unique effective action density $\mathscr{L}_{\text {eff }}$, for details see [23]:

$$
\mathscr{L}_{\text {eff }}\left[a_{\mu}\right]=\operatorname{tr}\left(\frac{1}{2} G_{\mu \nu} G_{\mu \nu}+\left(D_{\mu} \phi\right)^{2}+\frac{\Lambda^{6}}{\phi^{2}}\right),
$$

where $G_{\mu v}=\partial_{\mu} a_{v}-\partial_{\nu} a_{\mu}-i e\left[a_{\mu}, a_{v}\right] \equiv G_{\mu \nu}^{a} t_{a}$ denotes the field strength for the effective gauge field $a_{\mu}, D_{\mu} \phi=$ $\partial_{\mu} \phi-i e\left[a_{\mu}, \phi\right]$, and $e$ is the effective gauge coupling to be determined, see below. $\mathscr{L}_{\text {eff }}$ in Eq. (2) yields a highly accurate tree-level ground-state estimate and, as easily deduced in unitary gauge $\phi=2|\phi| t_{3}$, tree-level mass $m=$ $2 e|\phi|=2 e \sqrt{\frac{\Lambda^{3}}{2 \pi T}}$ for propagating gauge modes $a_{\mu}^{1,2}$ thus representing thermal quasiparticles. The emergence of mass in the propagation of effective plane waves relates to the collective effect of calorons residing in the thermal ground state, below we are interested in the induction of effective and local vertices by single calorons.

Demanding Legendre transformations between thermodynamical quantities (pressure, energy density etc.), which are a consequence of the temperature independence of the parameters of the fundamental action (infinite resolution), on the level of free thermal quasiparticles 1 , one obtains the following evolution equation [15]

$$
\partial_{a} \lambda=-\frac{24 \lambda^{4} a}{(2 \pi)^{6}} \frac{D(2 a)}{1+\frac{24 \lambda^{3} a^{2}}{(2 \pi)^{6}} D(2 a)},
$$

where $\lambda \equiv \frac{2 \pi T}{\Lambda}, a \equiv \frac{m}{2 T}=2 \pi e \lambda^{-3 / 2}$, and $D(y) \equiv \int_{0}^{\infty} d x \frac{x^{2}}{\sqrt{x^{2}+y^{2}}} \frac{1}{\mathrm{e}^{\sqrt{x^{2}+y^{2}}}-1}$. For a high-temperature boundary condition $a\left(\lambda_{i}\right)=a_{i} \ll 1$, the solution to Eq. [3] is approximately given as [23]

$$
a(\lambda)=4 \sqrt{2} \pi^{2} \lambda^{-3 / 2}\left(1-\frac{\lambda}{\lambda_{i}}\left[1-\frac{a_{i}^{2} \lambda_{i}^{3}}{32 \pi^{4}}\right]\right)^{1 / 2}
$$

\footnotetext{
${ }^{1}$ In the physical unitary-Coulomb gauge $\phi=2|\phi| t_{3}, \partial_{i} a_{i}^{3}=0$ the off-shellness of quantum fluctuations in the field $a_{\mu}$ is constrained by the maximal resolution $|\phi|$. Their contribution turns out to be negligible compared to that of thermal fluctuations [15].
} 
Thus for sufficiently low $\lambda_{i}$ function $a(\lambda)$ runs into the attractor $a(\lambda)=4 \sqrt{2} \pi^{2} \lambda^{-3 / 2}$. Using $a \equiv \frac{m}{2 T}=2 \pi e \lambda^{-3 / 2}$, this attractor corresponds to the plateau $e \equiv \sqrt{8} \pi$. Lowering $\lambda$ beyond a point, where $a \ll 1$ holds, the true solution to Eq. (3) runs into a thin pole at $\lambda_{c}$ of the form $e(\lambda)=-4.59 \log \left(\lambda-\lambda_{c}\right)+18.42$ with $\lambda_{c}=13.87$ [23]. The logarithmic pole of $e$ at $\lambda_{c}$ initiates a transition to a phase where thermal quasiparticles decouple (relevant for a higgsfree breaking of electroweak gauge symmetry [23]), the ground state starts to rearrange into a condensate of magnetic monopoles, and the formerly massless gauge mode picks up mass by the dual Meissner effect [15]. We conclude that almost everywhere in the deconfining phase the effective coupling assumes the constant value $e \equiv \sqrt{8} \pi$.

Let us now present the usual argument for counting the powers in $\hbar$ associated with vertices in the effective theory. We work in units where $k_{B}=c=\varepsilon_{0}=\mu_{0}=1$ but $\hbar$ is re-instated as an action. (So far we have worked in supernatural units $\hbar=c=k_{B}=1$ ). The (dimensionless) exponential

$$
-\frac{\int_{0}^{\beta} d \tau d^{3} x \mathscr{L}_{\text {eff }}^{\prime}\left[a_{\mu}\right]}{\hbar}
$$

in the weight belonging to fluctuating fields in the partition function can, in unitary gauge, be re-cast as

$$
-\int_{0}^{\beta} d \tau d^{3} x \operatorname{tr}\left(\frac{1}{2}\left(\partial_{\mu} \tilde{a}_{v}-\partial_{v} \tilde{a}_{\mu}-i e \sqrt{\hbar}\left[\tilde{a}_{\mu}, \tilde{a}_{v}\right]\right)^{2}-e^{2} \hbar\left[\tilde{a}_{\mu}, \tilde{\phi}\right]^{2}\right),
$$

where $\tilde{a}_{\mu} \equiv a_{\mu} / \sqrt{\hbar}$ and $\tilde{\phi} \equiv \phi / \sqrt{\hbar}$ are assumed indepent of $\hbar$ [34], see also [35]. Notice that because of the terms $\propto \hbar^{0}$ in (6) the unit of $\tilde{a}_{\mu}$ is length ${ }^{-1}$. Thus the coupling $e$ must have the unit of $1 / \sqrt{\hbar}$ (and the unit of $\tilde{\phi}$ is also length ${ }^{-1}$ ). As a result, we have $e \equiv \frac{\sqrt{8} \pi}{\sqrt{\hbar}}$ almost everywhere in the deconfining phase. Because mainly (just-not-resolved) calorons with $\rho \sim|\phi|^{-1}$ contribute to the effective theory, the action $S_{\rho \sim|\phi|^{-1}}$ of each such calorons reads

$$
S_{\rho \sim|\phi|^{-1}}=\frac{8 \pi^{2}}{e^{2}}=\hbar
$$

Equation (7) has implications:

First, it states that Planck's quantum of action $\hbar$, whose introduction in (5) ultimately has its origin in the laws of Quantum Mechanics and which accounts for quantum corrections (number of loops) in the effective theory, coincides with the Euclidean action of a just-not-resolved selfdual (classical) field configuration. That is, the reason why $\hbar$ really is a constant can be traced to the universal constancy of $e$ (almost no dependence on $T$ and on the specific Yang-Mills scale $\Lambda$ of a given $\mathrm{SU}(2)$ gauge theory) and the fact that there is one unit of conserved topological charge to a caloron. Moreover, rather than multiplying first and second powers of $\hbar^{1 / 2}$ onto the powers $e$ and $e^{2}$ of an a priori unknown coupling constant $e$ appearing in the three- and four-vertices of a Yang-Mills theory we would infer that these vertices are induced by just-not-resolved individual calorons.

Second, for the fine-structure constant $\alpha$ of Quantum Electrodynamics (QED) to be dimensionless,

$$
\alpha=N^{-1} \frac{g^{2}}{4 \pi \hbar},
$$

the coupling $g$ in Eq. (8) must have the unit of $\sqrt{\hbar}$. $\left(N^{-1}\right.$ denotes a numerical factor that should relate to the mixing of the massless modes belonging to several SU(2) groups, see [16].) This happens if $g$ is taken to be the electricmagnetically dual coupling to $e$ :

$$
g=\frac{4 \pi}{e} \propto \sqrt{\hbar} .
$$

That is, a magnetic monopole liberated by the dissociation of a large-holonomy SU(2) caloron and other incarnations of this magnetic charge in the preconfining and confining phasel are interpreted as electric charges in the real world. Third, by virtue of $e \equiv \frac{\sqrt{8} \pi}{\sqrt{\hbar}}$ the factors $e \sqrt{\hbar}$ and $e^{2} \hbar$ in (6) do not depend on $\hbar$. Thus, by the above assumption that the fields $\tilde{a}_{\mu}, \tilde{\phi}$ are independent of $\hbar$, the weight (5) is also independent of $\hbar$, and thus the effective loop expansion is not an expansion in powers of $\hbar$. This is in contrast to renormalized perturbation theory where the value of the coupling is assumed to be independent of $\hbar$.

\footnotetext{
${ }^{2}$ For example, the isolated charge situated in the center of the flux eddy associated with the locus of selfintersection of a center-vortex loop in the confining phase [23, 36].
} 


\section{MOMENTUM TRANSFERS IN AN EFFECTIVE 4-VERTEX}

According to the Feynman rules associated with the effective theory (2) the 4-vertex is local and reads

$$
\begin{aligned}
\Gamma_{[4] a b c d}^{\mu v \rho \delta}= & -i e^{2}(2 \pi)^{4} \delta(p+q+s+r)\left[\varepsilon_{f a b} \varepsilon_{f c d}\left(g^{\mu \rho} g^{v \sigma}-g^{\mu \sigma} g^{v \rho}\right)+\right. \\
& \left.\varepsilon_{f a c} \varepsilon_{f d b}\left(g^{\mu \sigma} g^{\rho v}-g^{\mu v} g^{\rho \sigma}\right)+\varepsilon_{f a d} \varepsilon_{f b c}\left(g^{\mu v} g^{\sigma \rho}-g^{\mu \rho} g^{\sigma v}\right)\right],
\end{aligned}
$$

where $\varepsilon_{a b c}$ are the structure constants of SU(2). We work in physical unitary-Coulomb gauge and in a real-time reformulation of the thermodynamics [23]. A $2 \rightarrow 2$ effective amplitude, where $\Gamma_{[4] a b c d}^{\mu \nu \rho \delta}$ links incoming four-momenta $p_{1}$ and $p_{2}$ with outgoing four-momenta $p_{3}$ and $p_{4}=p_{1}+p_{2}-p_{3}$, contains all fundamental scattering channels $s=\left(p_{1}+p_{2}\right)^{2}, t=\left(p_{3}-p_{1}\right)^{2}$, and $u=\left(p_{2}-p_{3}\right)^{2}$. This is expressed by the fact that the according effective treediagram subject to the local vertex $\Gamma_{[4] a b c d}^{\mu v \rho \delta}$ is invariant under permutations of the external legs. For the inducing caloron to remain unresolved in the effective theory we need to demand that $s, t, u \leq|\phi|^{2}$. Notice that for (naive) onshellness, either $p_{1}^{2}=m^{2}, p_{2}^{2}=m^{2}, p_{3}^{2}=m^{2}, p_{4}^{2}=m^{2}$ or $p_{1}^{2}=m^{2}, p_{2}^{2}=m^{2}, p_{3}^{2}=0, p_{4}^{2}=0$, the conditions $s \leq|\phi|^{2}$, $t \leq|\phi|^{2}$, and $u \leq|\phi|^{2}$ cannot be satisfied simultaneously 3 . A channel $s$ or $t$ or $u$ is called trivial if $s=0$ or $t=0$ or $u=0$, respectively. Let $N=1 \cdots 3$ be the number of nontrivial channels. Writing $\Gamma_{[4] a b c d}^{\mu v \rho \delta}=\frac{1}{N}\left(\left.\Gamma_{[4] a b c d}^{\mu v \rho \delta}\right|_{s}+\cdots+\left.\Gamma_{[4] a b c d}^{\mu v \rho \delta}\right|_{u}\right)$, we associate a nontrivial channel with each summand and assign the according constraint. For example, in the 4-vertex diagram for the one-loop polarization tensor of the massless mode ( $p$ the external and $k$ the loop momentum) the $t$ channel is trivial and thus $N=2$. Also, if $s \leq|\phi|^{2}$ for a given combination $p$ and $k$ then $u>|\phi|^{2}$ thus excluding the $u$-channel. Alternatively, $u \leq|\phi|^{2}$ for $p$ and $-k$ and then $s>|\phi|^{2}$ thus excluding the $s$-channel. Since the integrand, associated with the $k$-loop, is invariant under $k \rightarrow-k$ one effectively has

$$
\Gamma_{[4] a b c d}^{\mu v \rho \delta}=\frac{1}{2}\left(\left.\Gamma_{[4] a b c d}^{\mu v \rho \delta}\right|_{s}+\left.\Gamma_{[4] a b c d}^{\mu v \rho \delta}\right|_{u}\right)=\left.\Gamma_{[4] a b c d}^{\mu \nu \rho \delta}\right|_{s} .
$$

Eq. (11) holds equally well for the computation of two-loop corrections to the pressure that invoke one 4-vertex. (Here the two cases $p, k$ and $-p,-k$, where the $s$-channel constraint is satisfied (violated), are complemented by the two cases $-p, k$ and $p,-k$, where the $u$-channel constraint is violated (satisfied) while the loop-integrands are invariant under $p \rightarrow-p$ and $k \rightarrow-k$.)

\section{SUMMARY}

In this talk we have argued on thermodynamic grounds for a representation of Planck's quantum of action $\hbar$ in terms of the Euclidean action of an isolated, just not resolved SU(2) Yang-Mills caloron of topological charge unity. Such a caloron acts as an inducer of a local 3-vertex or 4-vertex in the effective theory. We have also re-discussed how the momentum transfer in a $2 \rightarrow 2$ amplitude, conveyed by such a caloron, is to be treated in the effective theory where the accoding Feynman rule is blind towards changes of the scattering channel.

\section{ACKNOWLEDGMENTS}

The author would like to thank Markus Schwarz and Niko Krasowkski for discussions.

\section{REFERENCES}

1. M. F. Atiyah, N. J. Hitchin, V. G. Drinfeld, Yu. I. Manin, Phys. Lett. A, 65, 185 (1978).

2. W. Nahm, Phys. Lett. B, 90, 413 (1980).

\footnotetext{
3 The author would like to thank Niko Krasowski for pointing this out.
} 
3. W. Nahm, All self-dual multimonopoles for arbitrary gauge groups, CERN preprint TH-3172, (1981).

4. W. Nahm, Self-dual monopoles and calorons in Trieste Group Theor. Method 1983, p. 189, (1983).

5. V. F. R. Jones, Bull. Amer. Math. Soc. 12, 103 (1985).

6. E. Witten, Commun. Math. Phys. 121, 351 (1989).

7. S. K. Donaldson and P. B. Kronheimer, The Geometry of Four-Manifolds (Clarendon Press, Oxford), (1990).

8. G. 't Hooft, Nucl. Phys. B, 79, 276, (1974).

9. A. M. Polyakov, JETP Lett., 20, 194, (1974).

10. M. K. Prasad and C. M. Sommerfield, Phys. Rev. Lett., 35, 760, (1975).

11. B. Julia and A. Zee, Phys. Rev. D, 11, 2227, (1975).

12. N. Manton, Multimonopole dynamics in Monopoles in Quantum Field Theory (Word Scientific, Singapore), (1982).

13. M. Atiyah and N. Hitchin, The geometry and dynamics of magnetic monopoles (Princeton University Press, Princeton), (1988).

14. U. Herbst and R. Hofmann, ISRN High Energy Physics 2012, Article ID 373121 (2012).

15. R. Hofmann, Int. J. Mod. Phys. A 20, 4123 (2005); Erratum-ibid. A 21, 6515 (2006).

16. F. Giacosa and R. Hofmann, Eur. Phys. J. C bf 50, 635 (2007).

17. F. Giacosa and R. Hofmann, Progr. Theor. Phys., 118, 759, (2007).

18. M. Schwarz, R. Hofmann, and F. Giacosa, Int. J. Mod. Phys. A 22, 1213 (2007).

19. J. Ludescher and R. Hofmann, Ann. Phys. 18, 271 (2009).

20. R. Hofmann, Ann. Phys. 18, 634 (2009).

21. J. Ludescher et al., Ann. d. Phys., 19, 102 (2010).

22. C. Falquez, R. Hofmann, and T. Baumbach, Ann. d. Phys., 522, 904 (2010).

23. R. Hofmann, The thermodynamics of Quantum Yang-Mills theory: Theory and Applications, (Word Scientific, Singapore), (2012).

24. M. Schwarz, T, talk at ICNAAM 2012, symposium on Analysis of Quantum Field Theory II.

25. B. J. Harrington and H. K. Shepard, Phys. Rev. D 17, 2122 (1978).

26. K. Lee and C. Lu, Phys. Rev. D 58, 025011-1 (1998).

27. T. C. Kraan and P. Van Baal, Phys. Lett. B 428, 268 (1998).

T. C. Kraan and P. Van Baal, Nucl. Phys. B 533, 627 (1998).

28. D. Diakonov et al., Phys. Rev. D 70, 036003 (2004).

29. G. 't Hooft, Nucl. Phys. B 33, 173 (1971).

G. 't Hooft and M. Veltman, Nucl. Phys. B 44, 189 (1972).

G. 't Hooft and M. Veltman, Nucl. Phys. B, 50, 318 (1972).

30. B. W. Lee and J. Zinn-Justin, Phys. Rev. D 5, 3121 (1972).

31. G. 't Hooft, Phys. Rev. D, 14, 3432 (1976); Erratum-ibid. Phys. Rev. D, 18, 2199 (1978).

32. R. Jackiw and C. Rebbi, Phys. Rev. D, 14, 517 (1976).

33. R. Hofmann and D. Kaviani, arXiv:1204.4112 [gen-physics].

34. S. J. Brodsky and P. Hoyer, Phys. Rev. D 83, 045026 (2011).

35. J. Iliopoulos, C. Itzykson, and A. Martin, Rev. Mod. Phys. 47, 165 (1975).

J. F. Donoghue et al., Phys. Lett. B 529, 132 (2002).

N. E. J. Bjerrum-Bohr, J. F. Donoghue, and B. R. Holstein, Phys. Rev. D 68, 084005 (2003). [Erratum-ibid. D 71, 069904 (2005)]

B. R. Holstein and J. F. Donoghue, Phys. Rev. Lett. 93, 201602 (2004).

36. J. Moosmann and R. Hofmann, ISRN Math. Phys. 2012, Article ID 236783, (2012). 